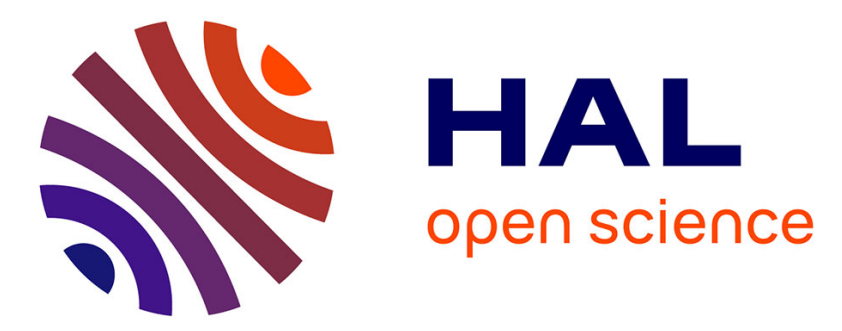

\title{
Puffy: A Mobile Inflatable Interactive Companion for Children with Neurodevelopmental Disorder
}

\author{
Franca Garzotto, Mirko Gelsomini, Yosuke Kinoe
}

\section{To cite this version:}

Franca Garzotto, Mirko Gelsomini, Yosuke Kinoe. Puffy: A Mobile Inflatable Interactive Companion for Children with Neurodevelopmental Disorder. 16th IFIP Conference on Human-Computer Interaction (INTERACT), Sep 2017, Bombay, India. pp.467-492, 10.1007/978-3-319-67684-5_29 . hal-01678515

\section{HAL Id: hal-01678515 \\ https://hal.inria.fr/hal-01678515}

Submitted on 9 Jan 2018

HAL is a multi-disciplinary open access archive for the deposit and dissemination of scientific research documents, whether they are published or not. The documents may come from teaching and research institutions in France or abroad, or from public or private research centers.
L'archive ouverte pluridisciplinaire HAL, est destinée au dépôt et à la diffusion de documents scientifiques de niveau recherche, publiés ou non, émanant des établissements d'enseignement et de recherche français ou étrangers, des laboratoires publics ou privés. 


\title{
Puffy: a Mobile Inflatable Interactive Companion for Children with Neurodevelopmental Disorder
}

\author{
Franca Garzotto ${ }^{1}$, Mirko Gelsomini ${ }^{2}$, and Yosuke Kinoe ${ }^{3}$ \\ ${ }^{1}$ franca.garzotto@polimi.it, ${ }^{2}$ mirko.gelsomini@polimi.it, ${ }^{3}$ kinoe@hosei.ac.jp \\ ${ }^{1,2}$ Politecnico di Milano - Dept. of Information, Electronics and Bioengineering, Milan, Italy \\ ${ }^{3}$ Hosei University, Department of Intercultural Communication, Tokyo, Japan
}

\begin{abstract}
Puffy is a robotic companion that has been designed in cooperation with a team of therapists and special educators as a learning \& play companion for children with Neurodevelopmental Disorder (NDD). Puffy has a combination of features that support multisensory stimuli and multimodal interaction and make this robot unique with respect to existing robotic devices used for children with NDD. The egg-shaped body of Puffy is inflatable, soft, and mobile. Puffy can interpret child's gestures and movements, facial expressions and emotions; it communicates with the child using voice, lights and projections embedded in its body, as well as movements in space. The paper discusses the principles and requirements underlying the design of Puffy. They take into account the characteristics of NDD and the special needs of children with disorders in the NDD spectrum, and provide guidelines for designers and developers who work in socially assistive robotics for this target group. We also compare Puffy against 21 existing commercial or research robots that have been used with NDD children, and briefly report a preliminary evaluation of our robot.
\end{abstract}

Keywords. Neurodevelopmental Disorder; Children; Inflatable Robot; Soft Robotics; Socially Assistive Robotics; Autism; Down Syndrome; Intellectual Disability

\section{INTRODUCTION}

Neurodevelopmental Disorder (NDD) is an umbrella term for a group of disabilities with onset in the developmental period that vary from specific limitations of learning and control of executive functions to deficits in social skills and intelligence, affecting personal, social, academic or occupational functioning [1].

Several researchers highlight the importance of early interventions to mitigate the NDD effects on the person's life and pinpoint how interactive technology can help in this respect. Particularly, the use of interactive robots has been proven promising in this arena and a number of studies have investigated the potential of these technologies (particularly for subjects with autism) to help children with NDD to develop cognitive, motor, and social skills. Still, given the wide range of NDD conditions and the specific characteristics of each single subject, there is space for new exploratory experiences involving robotic interaction to identify new forms of therapeutic and educational interventions for this target group.

The paper presents an innovative robotic companion called Puffy that has been designed as a learning \& play tool for children with different forms of NDD. Puffy is the latest outcome of a set of robotic companions that we have developed for this target group in cooperation with a team of NDD specialists, and evaluated at different therapeutic and education contexts [9][10][19]. Puffy engages users in free play and in game-based goal-oriented tasks, e.g., to promote imagination, communication, memory, or space and body awareness. Puffy has a combination of features that makes it unique with respect to existing robotic devices for children with NDD. It is mobile and has an egg-shaped inflatable soft body. It supports multimodal interaction, interpreting its body manipulation as well as user gestures, movements, facial expressions and emotions. It provides multisensory stimuli through voice, lights and projections embedded in its body, and movements in space. After a review of the state of the relevant literature (section 2), we describe some general requirements for the design of robots devoted to children with NDD (section 3) that offer guidelines for 
designers and developers who work in socially assistive robotics for this target group. The requirements take into account the characteristics of NDD and are grounded on the state of the art, the lessons learned from our previous projects, and the feedbacks from the NDD specialists who have collaborated in the development of Puffy. We then discuss the design of our robot (section 4) and how it can be used in educational and therapeutic contexts (section 5). After presenting the enabling technology of (section 6), we evaluate Puffy from two perspectives. In section 7 we compare Puffy against 21 existing robots used for subjects with NDD and highlight the originality and completeness of our robot. This analysis exploits an evaluation schema that is based on the requirements discussed in section 2 and can be used as benchmarking framework for comparing future robots in this field. In section 8 we report a preliminary evaluation of Puffy involving NDD specialists and two children with NDD. Section 9 draws the conclusions and depicts the future directions of our research.

\section{RELATED WORK}

The review in this section focuses on Socially Assistive Robotics (SAR) and inflatable robots in relationship to NDD. While there is a wide number of study that explore the benefits of SAR in general, and some specific SAR features in particular, for subjects with $\mathrm{NDD}$, the application of inflatable robots for this target group is unexplored.

\subsection{SAR and NDD}

Socially assistive robots are characterized by the capability of communicating and interacting with users in a social and engaging manner [25] [68]. In the last years, many researchers have investigated their application for NDD subjects, mainly considering children with ASD - Autism Spectrum Disorder (e.g., [15][16][21][22][23][39][47][54][60] [64][68]). In contrast to other devices such as computers, tablets, and smartphones, socially assistive robots can play, learn and engage with children in the real world physically, socially and emotionally, and offer unique opportunities of guided, personalized, and controlled social interaction for specific learning purposes [67]. Many of the socially assistive robots used in NDD therapy are remotely controlled by caregivers [39] [40] [64]. Autonomous behavior is implemented only in few cases, to support a single type of tasks and to achieve a specific therapeutic goal [59] [TEO] such as imitation, communication or question answering. Autonomous socially assistive robots have been used successfully to attract attention, stimulate imitation, and improve communication, socialization, and behavioral skills [9] [38] needed for independent living.

\subsection{Socially Assistive Robots: Body Shape and Mobility}

Several researches explore the shape and movement capability of robots in relationship to NDD. Different shapes have been explored, from abstract ones to cartoon-like, simplified humanoids, or realistic human-like faces [15]. For example, the shape of Teo [9], a robot designed specifically for children with autism, resembles the popular cartoon characters of Minions or Barbapapà. Results from several researches pinpoint that individuals with NDD show a preference for something that is clearly "artificial" with respect to agents that have human-inspired characteristics [20] [58]. The research reported in [6] and [51] shows that subjects with NDD may respond faster when cued by robotic movement than human movement, and some socially assistive robots used in NDD therapy can move body parts [39] [60] [65] [66]. Mobility in the physical environment offers opportunities to engage children in space-related tasks. IROMEC [59] and Labo-1 [21] for example are mobile differential drive robots but their movements are slow and clumsy movements so that children may lose attention in the interaction. QUEBall [63] has a simple spherical morphology of a relatively small dimension and rolls while moving. It provides multiple visual and 
sound effects to encourage the child to play but, to enabling rolling, it offers limited tactile affordances and stimuli. Teo [10] includes a holonomic (omnidirectional) base that enables it to move at a speed of up to $1.2 \mathrm{~m} / \mathrm{sec}$ in any direction, which resembles the mobility capability of human beings. Teo supports space-related game tasks involving "joint" (robot + child) movements in the space which exploit the infrared and sonar distance sensors embedded in the robot body and an external depth sensor (Kinect).

\subsection{Socially Assistive Robots: Emotional Features}

Several SAR systems exploit emotional features that seem to benefit children with NDD. Keepon [39] [40] is a creature-like robot that is only capable of expressing its attention (directing its gaze) and emotions (pleasure and excitement). The empirical studies with autistic children showed that the robot triggered a sense of curiosity and security, and the subjects spontaneously approached Keepon and engaged in dyadic interaction with it, which then extended to triadic interactions where they exchanged with adult caregiver's pleasure and surprise they found in Keepon. KISMET ([12]) is an emotional robot which possesses eyebrows, ears, and mouth and expresses emotions depending on the way a human interacts with the robot. The robot's emotional behavior is designed to generate an analogous social interaction for a robot-human dyad as for an infant-caretaker dyad. Teo [10] supports users' emotional manifestation through the personalization of the robot. It is equipped with a set of a detachable pieces like eyes, eyelids, or mouths that can be attached to its body and enables children to create face expressions. In addition, as Teo's sensorized body can distinguish among caresses, hugs, and two levels of violent punches or slaps, the robot can react emotionally to different manipulations, using light, sound, vibrations, and movement to express different emotional states - happiness, angriness, or fear.

\subsection{Inflatable robots}

Inflatable robots [43] [52] [69] have recently received the interest of the research community as they have several advantages over rigid robots. A soft lightweight inflatable body contributes the increased safety and robustness. It is less likely to cause harm to humans during interaction and works as a shock absorber in a case of an accidental collision or an unexpected fall [4], protecting embedded sensors and devices from a potential damage. The use of inflatable robots has been investigated in some critical environments such as disaster relief and field exploration [43]. To our knowledge, their application to children's learning and play is unexplored. Still, inflatable robots have a potential for children with NDD as they meet the requirements for robustness and safety that are needed, and their deformable structures offer manipulation experiences that can be particularly engaging.

\section{DESIGN REQUIREMENTS}

The requirements $(R s)$ that informed the design of Puffy are grounded on several design guidelines that are available in the literature about robots for autistic children [15], [31], [47]. Starting from the analysis of the state of the art, existing design principles have been filtered, revised, and enriched to address the broader target of children with NDD. We also capitalize on our own prior experience in assistive technology for children with NDD [9] $[10][19][71][72][73]$ and on the collaboration with therapists and special educators specialized in NDD who have been collaborating in our past research and in the design of Puffy. A general consideration that pervades many of the requirements described below concerns the characteristics of the sensory and perceptual system of most children with NDD, and how these affect functioning and behavior. Subjects with NDD often have an abnormal capability of sensory discrimination (the ability of focusing on, and discriminating between, certain stimuli) and sensory integration (the ability of processing and properly interpreting multiple sensory signals at the same time). These deficits are thought to be one 
of the main reasons for irritability, difficulty in selective and sustained attention, or hyperactivity that characterize many subjects with NDD; They generate discomfort, frustration, and disengagement, and make enormously difficult to accept, express and interpret emotions, and to sustain social relationships. These deficits are also thought to originate functional deficits related to own body awareness and space awareness, elementary mechanisms of abstraction and generalization, problem solving, planning, and language [18] [62].

\subsection{Visual Appearance (R1)}

As most of children with NDD are visual learners and have frequent loss of attention, the visual appearance of the robot body is fundamental in the experience with the robot. The robot body is a mean to attract the child's attention and engage her in an experience, as well as to communicate and convey meaning. Considering the sensory problems described above and the consequent risk of distress, the robot should avoid visual overstimulation, as the one created by different brightly colored body parts and aggregation of (moving) components of different shapes [31] [61]. Few neutral colors should be used for the body and harmonized to promote relaxation and trust [35]. Their visual attributes should be functional to their affordance and to the goal they are meant to support during interaction. The use and amount of different colors should be carefully calibrated considering the sensorial characteristics of each child and different visual configurations should be available for the same task. The shape of the robot should evoke a familiar element, possibly something that the subject likes such as cartoon characters. Considering Mori's conjecture about the uncanny valley [48], and the difficulty of NDD subjects to interpret the multiple signals expressed by the complexity of human faces and body, abstract minimalistic "harmonic" shapes are thought to be preferable to realistic representations (e.g., human like) [58] [61]. Still, it is important that some facial components are included, particularly eyes, so that the child could easily understand where the robot is facing and "looking at", and establish eye contact with the robot. Children with NDD (especially those with autism) are uncomfortable in making eye contact with humans, and simulating eye contact with the robot might help them to generalize this concept in human-human relationships [57]. To facilitate eye contact with the robot, the most appropriate size for the robot should correspond to the average size of the target group [10][57][60].

\subsection{Multimodality (R2)}

Multimodality, i.e., the provision of different interaction modes that involve different (sets of) skills and sensory stimuli, have a number of advantages for children with NDD. Supporting multiple modes of interacting with the robot opens up opportunities to engage the children in different ways, each one focused on specific and evolving learning needs, and to promote cause-effect understanding skills which derive from experiencing the action-feedback loop in different situations. Still, multimodality involves some potential risks for this target group. The perceptual experience of subjects with NDD is often abnormal and these persons may have impairments in filtering or processing simultaneous channels of visual, auditory and tactile inputs. They may perform poorly across different interaction modes and during conditions that require processing different requests and stimuli. The robot should offer a gamut of "single mode" interactions that can be used one by one, and also enable progressive combinations or concatenations of different interaction modes. These must be carefully calibrated so to enable children to practice tasks at the proper level of sensory complexity and to master the difficulty of perceiving inputs across multiple modes. According to the current literature, the interaction modes that have been proved effective for children with NDD are spatial, tangible, vocal, and emotional. 


\subsection{Spatial Interaction (R3)}

Spatial interaction exploits how humans use the space to regulate the reactive behavior of an interactive technological artifact, and has recently been emphasized as one of the important design aspect of a social assistive robot [45], [49], [55]. Spatial interaction requires that the robot is able to move as naturally as possible in the space, to sense and interpret the user position, orientation, movement, direction, and to react consistently. Spatial interaction help children with NDD to learn spatial awareness, i.e., the ability to be aware of oneself in space, which is often weak in these subjects. Spatial awareness requires the creation of a contextualized body schema representation - a sense of where your body is in the space, and involves the understanding of the relationship between physical objects and oneself in the space, both when objects are static and when they change position, learning concepts like distance, speed, "near", "behind", and similar.

\subsection{Tangible Interaction (R4)}

Manipulation and tactile experiences plays a fundamental role in the development of sensory-motor capabilities as well as cognitive functions [70]. Several therapeutic tasks for children with NDD involve touch and manipulation of physical materials as a means to improve the capability to interpret stimuli processed by the tactile system and to improve own-body awareness. Similar skills can be promoted in a playful, safe, and controlled way through tangible interaction with the robot, enabling touch and physical manipulation of its body associated to consistent feedbacks. Physical touch is also one of the most basic, but at the same time most important forms of human communication. Through physical interaction with the robot children with NDD can learn to express and interpret this form of communication. Particularly, while an inappropriate tactile interaction with a human (e.g., pushing) would lead to a negative reaction which can be enormously frustrating for subjects with NDD, the similar action on a robot can trigger stimuli that help them reflect on their behavior and build a sense of stability and confidence [56]. Tactile interaction involving the feature of being "huggable" is recently emphasized as one of important aspects in the design of Robot Assisted Therapy [20] [68] [9][10].

\subsection{Vocal Interaction (R5)}

Children with NDD may have deficits in speech production and understanding - a complicated process involving the coordination of motor, auditory, somatosensory systems and several cognitive functions. Many therapy programs for these subjects include activities to help them communicate verbally in a useful and practical way. A robot equipped with vocal interaction can support vocalization capability and in principle be integrated in existing speech-therapy treatments. The controlled study reported in [24] show more positive effects on vocalization and speech capability when the considered robot interacted through remotely arm, ears, mouth, and eye movements and speech (remotely generated and controlled by the investigator) compared to the effects achieved when voice features were missing. Qualitative observations emerging from empirical studies on Teo [9] highlights that vocal interaction through reward phrases and songs (generated after a task completion) promotes engagement and fun.

\subsection{Emotional Interaction (R6)}

Emotional information exchange plays an important role in human-human interaction. Current SAR research considers emotional interaction one of the principal ways to achieve trust and believability [8], making the user feel that the robots "understand" and "care" about what happens in the world. In addition, a robot that can both manifest its emotional states and interpret users' emotions helps subjects with NDD to develop the capability of emotional information exchange, i.e., to learn how to interpret and manifest emotions, 
which is fundamental for human interpersonal communication. Emotions can be expressed through different signals depending on the actuation characteristics of the robot: different face expressions, music, voice tones, movements in space or body movement rhythms convey different emotional signal.

\subsection{Multisensory Feedback (R7)}

The action-feedback mechanism which is intrinsic in interaction promotes two basic and fundamental skills: cause-effect understanding and sense of agency, i.e., the feeling of being able to exercise control and of obtaining a coherent response. Once these skills are established, a person learns that (s)he can also affect different situations and people, and is motivated, for example, to use communication in its many forms (e.g., by requesting, questioning, or refusing) to manipulate situations. Reward stimuli, to acknowledge the correct completion of a multi-action task should also be explicitly acknowledged so that the child feels gratified for the achievement [61]. For example, lighting up part of the robot body or playing music or songs, showing animations is thought to be particularly engaging and encouraging [27] [47]. Still, for the reasons discussed at the beginning of this section, all stimuli must be carefully designed, and calibrated properly to avoid overstimulation and discomfort.

\subsection{Safety (R8)}

The robot should be "be harmless to patients physically, psychologically, and ethically" [53]. To this extent, sharp edges should be avoided favoring soft textures [47]. In addition, the robot body should be robust enough to reduce the effects of voluntary or involuntary disruptive actions, considering that children with NDD can be uncontrollably exuberant or impulsive at times.

\subsection{Configurability (R9)}

Because children with NDD have unique and evolving needs, the value of technology for this target is directly related to its ability to adapt to specific needs and learning requirements of each person or group. [13] [50]. The robot should be integrated with the possibility for therapists to configure it (e.g., increasing/decreasing/removing sensory stimuli, or changing visual and sound rewards) to adapt the interactive experience to the specific profile of the current user(s).

\subsection{Dyadic Execution Mode (R10)}

Involving children in robotic interaction requires a combination of remotely-controlled and autonomous modes. Remote control gives the complete control of the robotic behavior to the caregiver. This is important to manage situations created by children's unexpected actions, to unlock stereotyped behaviors (typical of children with autism), to create new stimuli in response to children's interactions with the robot or movements in the space, or to adapt the stimuli to the specific characteristics of each child. Still, remote control is demanding for the caregiver. She must pay a constant attention to the child and at the same time must operate on the robot, controlling it in a believable and timely way and giving the impression that the robot is behaving autonomously and consistently with the current context. This burden can be alleviated by including some autonomous behaviors in the robot, i.e., programmed $<$ stimuli-user action - stimuli $>$ loops or flows of loops that enable the robot to act in the environment and interact consistently with some a pre-define logic.

\subsection{Multiple Roles (R11)}

The robot plays different functional roles in the interaction with the children [15]: 
a) Feedback: it reacts to an action performed by the child, promoting cause-effect understanding, and plays as rewarding agent, to offer positive reinforcement and promote selfesteem.

b) Facilitator: it suggests what to do and when to do it facilitating task execution

c) Prompt: it acts as a behavior eliciting agent enhancing attention and engagement

d) Emulator: it plays as an emulator (acts as the child) or something that is imitated, to trigger the child's imitative reaction and skills

e) Restrictor: it limits the child's spatial movement possibilities

f) Social Mediator: it mediates social interaction between the child and others (therapist, peers); it acts as a communication channel through which the child expresses her communication intents as well as a tangible material for shared activities

g) Affective and emotional agent: it facilitates the creation of affective bond between the robot and the child/children, helping subjects to unlock their emotional rigidity and to feel emotions; it stimulates the children's capability of manifesting their own emotions and interpreting the others' emotional expressions [70].

\section{THE DESIGN OF PUFFY}

Puffy has been co-designed done in partnership with a team of 2 designers, 4 engineers and 15 therapists (psychologists, neuro-psychiatrists, special educators) from two different rehabilitation centers. In what follows we describe the physical and behavioral characteristics of the robot matching them to the requirements discussed in the previous section.

\subsection{Physical Characteristics}

General shape: The visual appearance of Puffy (Figure 1) meet all requirements stated in $R 1$. Its white shape, externally made of a thin, white, opaque plastic textile is inspired by Baymax, the inflatable healthcare robot of the popular Disney animated movie Big Hero 6, and reminds a familiar character that children like and have positively experienced in everyday life. Puffy is approximately $130 \mathrm{~cm}$ high (the average height of our target group) and its only facial elements are two black eyes, to help user understanding what the robot is gazing at. Its wide round belly and its curved silhouette confer a fluffy and comfortable warm appearance and contributes to relax children, to promote affections and trust (R11.g), and to give the impression of playing as a gentle "big brother".

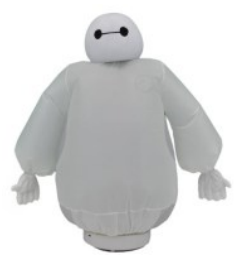

Figure 1. Puffy
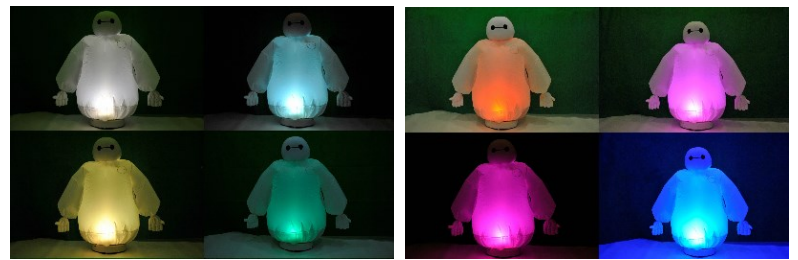

Figure 2. Light effects in Puffy

Dynamic inflatable structure: Puffy is characterized by an inflatable structure. An embedded fan (that also serves a cooling purpose) is used to blow up Puffy's light plastic "skin" at the beginning of a session and to dynamically transform the shape and size of its inflatable structure in order to convey emotional body signals. Puffy can simulate relaxed breathing (through rhythmic inflating-deflating), manifest satisfaction and confidence (expanding the body through inflation), or express discomfort and sadness (compressing the body by reducing the air inside), enforcing the robot role as Affective and Emotional Agent (R11.g). The resulting body - big, soft, flexible in shape, and humorously rounded - makes 
Puffy robust, safe and harmless (R8), and pleasurable to touch and hug (R4). These features facilitate the role of Puffy as "Social mediator" (R11.f). For example, Puffy can be manipulated by many children together at the same time (Figures 3-4-5).

Lights: Inside Puffy there is a commercial smart lamp (a Philips Hue Go) that generates a gamut of luminous feedbacks $(R 7)$ that, perceived across the white opaque fabric of the robot body, give a sense of magic (Figure 2). Light stimuli are used not only as an aesthetic medium to attract and create engagement (R11.c). Rhythmic dynamic light can complement the rhythmic expansion/compression of the robot's body, and increase the emotional effect (R11.g). As colors and intensity evoke and convey different emotions, luminous stimuli can be used to enhance emotional interaction (R6) and affective bond (R11.g).

Music and voice: Music not only offers stimuli for the sound sensory system $(R 7)$ but is also known to conveying emotions and affect wellbeing and emotional states (R6 and $R$ 10.g). In some phases of a therapeutic session such as during the introduction and the breaks, Puffy plays soft songs at the frequency of $432 \mathrm{~Hz}$ (a frequency that is acknowledged to influence heart rate and improve relaxation), to establish a pleasurable calming atmosphere. While children are engaged in a task, cheerful music offer rewards or is used to recapture user attention (R11.c). Puffy's cheerful voice (R5) offers vocal instructions and feedbacks during interaction and tasks execution, such as back-channeling expressions (" $\mathrm{mm} \mathrm{mm}$, uhmmm, $\mathrm{mm}$ mmmm...), reinforcement or reward phrases.

Vocal interaction capability can range from the production of voice instructions and vocal feedbacks (back-channeling expressions, reinforcement or reward phrases, songs, or rhymes) to the capability of interpreting the users' speech and reacting consistently.

Multimedia on-body projections: A compact projector is embedded inside Puffy's body and displays visual stimuli (images, videos, or animations) on its belly $(R 7)$. These contents - which in rigid socially assistive robots are displayed using a tablet, a PC screen, or a smartphone placed on the body - [38] provide instructions, suggestions, or feedbacks $($ R11.a-b) for the tasks in which the children are involved, or are associated to the story Puffy is telling (as discussed later in the paper). Thanks to the white opaque light weighted plastic material and the shape of the inflatable body, projections result curve-rounded and with ambiguous borders that create a pleasant visual effect and enhance the sense of magic of the story being told, contributing to increase the affective bond with the robot (R11.g).

Mobility: Puffy does not walk like Baymax character. Nevertheless, thanks to its holonomic base, our robot has a fluid mobility and is free of moving on the floor in any direction at a speed similar to that of humans in indoor environments (R3), wandering around, chasing the child, or getting close or faraway. Puffy's mobility features, described more precisely in the following section, support the robot's roles as Emulator (R11.d) and Restrictor (R11.e). Movements can be also used as prompts, to complement the prompting capability of music, voice, and projected visual contents (R 10.c).

\subsection{PUFFY: interaction and behavior}

Puffy-children interaction is multimodal (R2). Puffy supports tangible and vocal interaction ( $R 4$ and $R 5)$ - enabled by its capability of sensing and interpreting touch, sound, and speech, spatial interaction $(R 3)$ - enabled by the robot capability of moving and sensing users' movements, distance and position - and emotional interaction (R6) - enabled by the capability of recognizing the user facial expressions and voice signals, and to manifest emotional signals. Both tangible and spatial interaction can be combined with emotional interaction, as described below. 


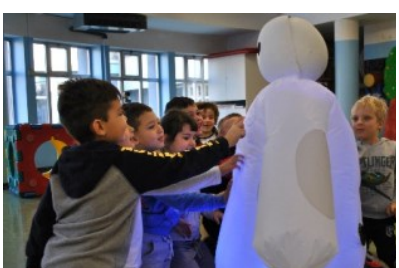

Figure 3. Shared Interaction with Puffy

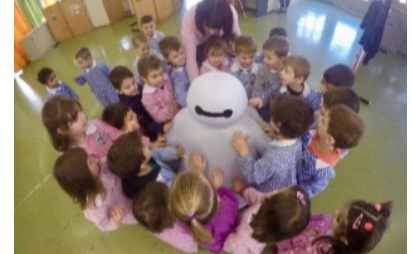

Figure 4. A group of children (two of them with NDD) playing together with Puffy at a local school

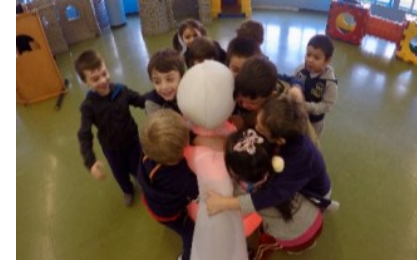

Figure 5. Manifestations of Affection towards Puffy

Coordinated Spatial + Emotional Interaction: Puffy senses and interprets the physical spatial relationship between itself and the children (e.g., relative position, movement, orientation), which enables various forms of spatial interaction. For example, if an educational session has just started and Puffy locates the child far away, the robot attempts to attract the child's attention: it emits some cheerful sound and turns left and right (as if it were looking around) while its body is illuminated with soft blue light rhythmically changing intensity to increase the sense of movement. Puffy can also combine spatial interaction with emotional interaction, taking into account the psychological and emotional aspects of the spatial relationship between itself and a child. To this end, Puffy exploits an Interactional Spatial Relationship Model (Figure 6) which considers the following elements:

(a) Interpersonal distance, measured by an embedded depth sensor and classified according to the Hall's zone system of proxemics interaction (e.g. public, social, personal, intimate [34]); the definition of the parameters for users preferred interpersonal distance can be customized according to various conditions including a child's age and the current task, e.g., as a competitive or cooperative situation [37]).

(b) Relative (child-robot) bodily orientations (e.g. face-to-face, side-by-side). Bodily orientation and interpersonal distance are a form of non-verbal communication that implicitly convey a child's current intention how a child wants to manage his/her relationship with Puffy [46]

(c) Child's and robot's movements in space, defined by physical parameters such as direction and speed. Depending on the context, the kinematic behavior is interpreted both as "functional" to the current task, or as a psychological and emotional cue (e.g., "escaping from the robot" may be interpreted as an action required by the game or a signal of discomfort)

(d) Child's emotional state, which is detected from the analysis of the child's voice tone [42] and facial expressions

(e) Child-robot eye contact, which is again based on the analysis of the child's image; as already mentioned (section 3.1) eye contact is an important nonverbal communication behavior to express interest, attention, and trust towards a conversation partner, and is often missing in children with NDD (especially those with autism),

(f) Robot's emotional state: the robot's current emotional state resulting from the Physical Manipulation by the children (see "Tangible") and from movement values.

The current status of the robot-child relationship, defined by the set of the above variables, is used for determining the robot spatial and emotional behavior, as in the following example. Let us assume that there is only one child interacting with Puffy and the model variables have the following values:

(a) Sinterpersonal distance $=140 \mathrm{~cm}$, i.e. located in a point of transition from "social" to "personal" distance $\}$

(b) \{relative bodily orientations: child $=0$, puffy $=0$, i.e. "face-to-face"\}

(c) \{child's spatial movement= "standing-still", i.e., no significant movement detected $\},\{r o-$ bot's spatial movement= "approaching to a child at velocity $0.20 \mathrm{M} / \mathrm{sec}$ "\}, 
(d) \{child's emotional state $=$ "mild", i.e., child's voice detected at medium level of loudness + smile recognized in the face $\}$

(e) \{child-robot eye contact = "eye-contact by a child detected", i.e., sufficient level of child's interest and attention towards the robot $\}$,

(f) $\{$ robot's emotional state $=$ "quiet", i.e., no previous hit; no movement, vibration, or blink $\}$.

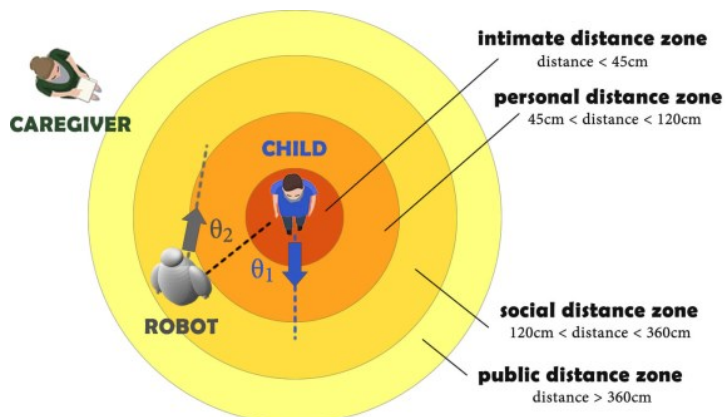

Figure 6. Model of Interactional Spatial Relationships

Based on the above analysis, Puffy decides that it is the appropriate timing to make a greeting, and sets its voice at medium-high level of loudness (as appropriate at the edge of social zone); then it continues moving towards a child only after it detects the absence of negative emotional response from the child. Approaching the "personal" distance zone around the child, Puffy re-evaluates the current status of the robot-child relationship and adapts its behavior by taking into account the potential risk of its current and next reactions.

For example, Puffy may estimate that it should slow down its speed. The "personal" distance zone around the child is typically reserved to friends and family members who know and trust each other [34], and to avoid the child's feeling of an undesirable intrusion of his personal space of the child), Puffy must be ready to stop. After the robot detects that there is no negative response from the child, it considers that it is the right time to start some form of explicit communication, and for example engages the subject in a conversation adjusting its voice level at medium- loudness (as appropriate at personal distance).

Tangible Interaction: Puffy employs two modes of tangible interaction: Physical Manipulation and Functional Touching. Physical Manipulation involves the tactile contact with Puffy (Figure 4) and involves elements of emotional interaction. The robot's sensorized body can distinguish among caresses, hugs, and two levels of violent punches or slaps, measuring the intensity and dynamics of the body deformation induced by the physical contact. Depending on the evaluation of the manipulation, Puffy produces a specific emotion-based behavior by employing a map of multimodal emotional expressions [41]. Puffy becomes Happy when its body is softly caressed or touched. It responds to this pleasurable manipulation by emitting sound expressions of pleasure, vibrating, and rotating itself cheerfully, while internal lights becomes green and blink slowly. Puffy becomes Angry when its body is hit with moderate force. It responds to this rude manipulation by moving sharply towards a child, turning light to red, inflating its body and growling (grrr). Puffy becomes Scared when its body is brutally hit. It reacts by slowly retreating itself saying "what a fear!" while lights become yellowish and pulse slowly and the body shape shrinks. Functional Touching allows a child to make a choice (Figure5), express simple instructions to Puffy, or answering a question, by pressing Puffy's body in a specific area. Using the embedded projector, the active areas for functional touching appear dynamically on Puffy's belly, recognizing the position of the child's touch. Digital contents can be personalized by therapists by inserting the tags that are more meaningful for the activity, either realistic 
images or PCSs (Picture Communication Symbols), commonly used in Augmented Alternative Communication interventions [28].

Voice Interaction: Puffy can react vocally to situations that change its emotional state, as discussed above. Knowing the current state of a task (e.g., "complete"), the robot can use voice to reward the children. In addition, Puffy can interpret at some degree the children's speech, namely, the main sentiment of children's vocalizations and the main concepts expressed, and is programmed to react consistently.

Execution Mode: The execution of Puffy's behaviors is performed in two modalities remotely controlled and autonomous agent (R10). In remotely controlled mode, the caregiver triggers the desired stimuli on Puffy's body and drives the robot movements using a remote controller (a joystick). As an autonomous agent, Puffy is preprogrammed to act autonomously according to the interaction rules defined in section 4.2 and the logic of the activities defined in section 5.2.

\subsection{Configurability}

Puffy's behaviors, interaction modalities and sequence or intensity of the stimuli offered are customizable by therapists to address the evolving needs and preferences of each specific child (R9). A simple control panel enables therapists to (1) assign each activity to each child; (2) setup a child's curriculum with a progressive set of levels; (3) add, remove and edit projected elements; (4) choose feedbacks and rewards such as onboard lights colors, voice, and multimedia stimuli.

\section{USING PUFFY IN EDUCATIONAL OR THERAPEUTIC CONTEXTS}

Puffy can be presented as a new play companion and exploits the potential of gamebased learning engaging children through two forms of play: free play and structured play. Both free and structured play can be performed by a single child or (preferably) in group, to enhance the social dimension of the experience (R11.f).

Free play: Free play consists of spontaneous, intrinsically motivated, unstructured tasks and has a fundamental role for the child's physical, cognitive and social development [14]. Free play with Puffy involves all interaction modes but functional touching. The children spontaneously manipulate the robot, try its physical affordances, and explore the physical space together with the robot, and the flow of stimuli is fully under children's control (with possible interplays of stimuli remotely triggered by the caregivers when needed). Free play can be used to facilitate the progressive mental and emotional states of relaxation [32], affection [2], and engagement [36], which are fundamental in any learning process of children with NDD and are a precondition for the execution of structured, goal oriented activities. Initially, the presence of Puffy in the playground could be potentially worrying, as children with NDD are often afraid of the unknown. They should learn that this "object" is predictable and safe, become confident that it is good, harmless, and inoffensive, and progressively achieve a state of relaxation, both in the relationship with the robot and towards the other children in the group. As the familiarization with the robot proceeds, children also develop the feeling of strong affective bond towards Puffy, i.e., affection, which facilitates a more persistent positive attitude towards the robot. Affection in turn is known to promote engagement, the state of active, voluntary involvement in an activity and the willingness to act upon the associated objects maintained for a relatively prolonged time.

Structured play: Structured play is focused on the development of specific skills by executing activities that follow a predefined flow of tasks and stimuli programmed in the Puffy. The types of activities developed so far are "Storytelling", "Choice" and "Tag".

Exploiting the general learning potential of storytelling for all young children [29], stories are widely used in educational and therapeutic practices for children with NDD to 
stimulate imagination, curiosity, and emotional development [33] [42]. Like other SARs for NDD [23] [42], Puffy is able to narrate stories (Figure 7). Prior to starting a storytelling, the robot attempts to establish the appropriate spatial relationship with a child by re-adjusting a suitable position that promotes a shared experience, while it continues monitoring the emotional response of a child. Puffy narrates stories by talking and projecting interactive multimedia contents on a curve-rounded screen of its belly. It can also perform movements and activate light and vibration effects to enhance the emotional effect and underline some moments or situations of the narration. In addition, Puffy can engage children during the story, e.g., asking questions (about events, characters, or places in the plot) or prompting interactions or movements, and consider children's actions and interpersonal spatial relationships to determine its own next behavior.

Choice games aim at developing the willingness and capability of making choices as well as memory skills. A game of this types starts with a projection on the robot body where multiple choices are available, then Puffy asks a question and invites children to respond by touching a projected button (Figure 7).

Tag games aim at fostering children's movement. For example, Puffy invites the children to play together and to move in the room, and then starts chasing them (Figure 8).

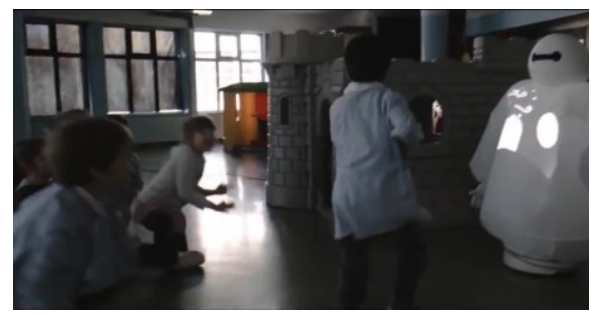

Figure 7. Puffy telling a story

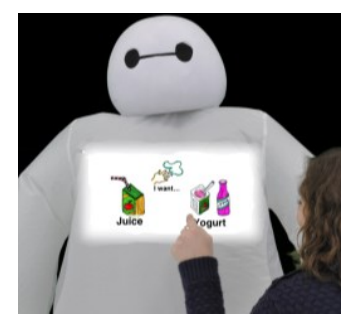

Figure 8. Choice game

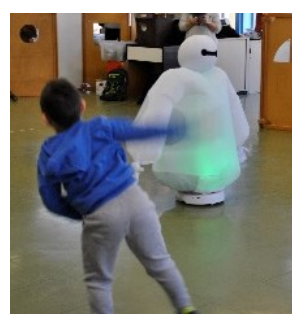

Figure 9. Tag game

\section{Caregivers' role}

As in any intervention for subjects with NDD, during both free and structured play with Puffy caregivers - educators or therapists are present to assist and monitor the children, and interact with them striking a balance between giving them autonomy and mediating the experience with the robot. The caregivers act as "facilitator", to suggest what to do and when to do it, as "prompt", to promote attention and concentration, and as "play companion", to share emotions, surprise, and fun. For example, they frequently talks with the children and asks (or explains) what happens to Puffy, and sometime help them also with gestures, e.g., moving a child's head or body in a specific direction when it is evident that (s)he has lost attention. The technological experience becomes an opportunity for social connection between children and caregivers, exposing subjects with NDD to verbal communication and stimulating them to "think aloud" and practice language, which are all important elements to develop appropriate social competences. Depending on children's behaviors and emotional reactions during play, may switch from autonomous to controlled mode, to trigger specific stimuli, suspend-replay a task, or activating a different one.

\section{TECHNOLOGY}

An embedded board (Arduino Uno) operates as general control and communication device. The board communicates with a wheeled robotic base (iRobot iCreate) placed on the bottom of the body, six Force Sensing Resistors (FSRs) and the fan. The embedded equipment includes a 
mini PC, which manages most of the computational aspects of the robot behavior and is connected both with the Arduino Uno and the Microsoft Kinect Sensor placed inside the robot's head. A skeleton sustains a projector, a Philips Hue Go light and the audio speaker (Figure 10).

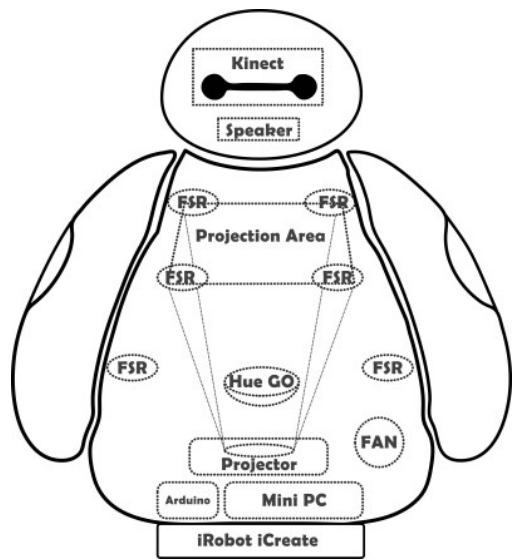

Figure 10. Puffy technology positioned inside the inflatable structure

The FSRs are used for tangible interaction. Four FSRs enable Functional Touch and are placed at the corners of the projection area, where the user would touch Puffy in order to make choices (Tag games). Two additional FSRs, placed in a lower position (approximately aside the arms, on the left and right side of the body) are used for Physical Manipulation. Using the values coming from these sensors and the time elapsed between one value and the following one (i.e., the duration of a manipulation with the same intensity), we define a set of value ranges associated to different types of Physical Manipulation e.g., caress (low force and low-medium duration time), hug (high force and long duration time), punch or slap (medium force, low duration time), violent punch or slap (high force, low duration time). A caress is recognized as a "soft" deformation of the FSR (force intensity between 400 and 600) that lasts for about $1000 \mathrm{~ms}$. A hug occurs when the deformation is approximately the double and lasts approximately $1500 \mathrm{~ms}$. A slap or a punch is detected when a big deformation of the FSR (force intensity $>900)$ occurs in a very short time $(<1000 \mathrm{~ms})$. Examples of manipulations data along the time are shown in Figure 11.

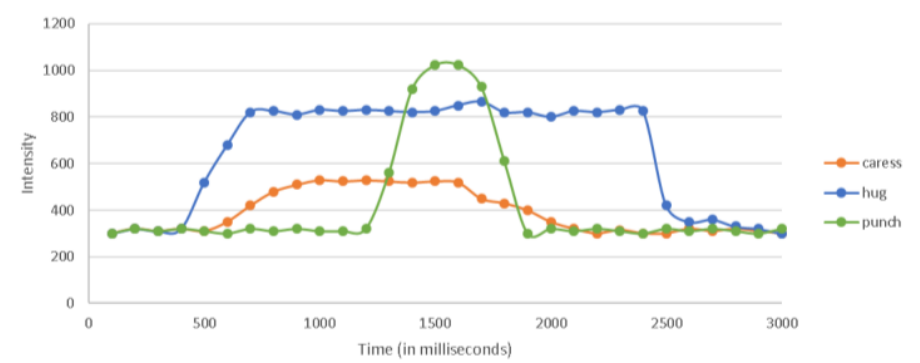

Figure 11. Examples of children's manipulations (caress vs hug vs punch) showing the Force intensity ( $\mathrm{Y}$ axis) sensed by FSEs along the Time ( $\mathrm{X}$ axis) in 3 different interactions with Puffy

Differently from other robotic companions employed for the care of NDD children (e.g. Teo [10]), it is Puffy in "first person" that evaluates the parameters needed to execute spatial interaction and determines its own behavior in response to children's movement, position, and orientation in space. The Microsoft Kinect placed in the robot head embeds a depth camera (which look like eyes in Puffy's face), a color camera, and an array of microphones. Depth and color 
cameras generate body skeleton data that are used to evaluate the following variables along the time, which are calculated for the child who is closest to the robot: relative Puffy-user position; Puffy's and user's body orientation; user's movement and direction. These variable are computed using standard Kinect technology (SDKs) as well as data coming the robot base ([71][72][73]). Examples of these variables are depicted in Figure 6 - section 4. In this figure, Theta_1 defines the breadth of the angle between the child's direction and the segment connecting the robot's and the child's position. Theta 2 is the breath of the angle defined by the robot's direction and the same segment. As discussed in section 4.2, the values of these variables, integrated with the results of speech and image processing (see below) are used by a rule-based Behavioral Engine to decide following behavior for the robot to perform. To recognize facial expressions, we use the stream of data generated by the Kinect color and Depth cameras for the child who is closest to the robot. The image processing and facial analysis algorithms use the Kinect SDK and the EmguCV library, a cross platform .Net wrapper to the OpenCV image processing library. OpenCV (Opensource Computer Vision) was selected for its computational efficiency and its strong focus on real time applications. The Kinect microphone array capture user's speech.

The user's speech is interpreted by a Speech Analyzer module, which considers the vocal input of the child who is closest to the robot, and exploits IBM Watson technology and OpenSmile. IBM Speech-to-Text API is used to convert speech into written text. IBM Natural Language Understanding API is used to analyze this text and extract relevant metadata (concepts, sentiment, and emotions). OpenSmile is an open-source and real-time sound feature extraction tool, which we use to automatically extract the tone and pitch of child's speech. Combining these information with the metadata, a rule-based component of the Behavioral Engine generates the textual answer that is converted in Puffy's voice using IBM Text-to-Speech API. The above process has a number of challenges, which are mainly related to group interaction. Particularly, the issue is the identification of the "closest child" and the need of filtering out his(her) data, permitting separation of this child's vocalizations and images from all other speech and other sounds that are produced within the immediate environment as well as from the images of other persons nearby. We are currently working to improve the level of accuracy reached so far.

\section{COMPARISON}

Puffy provides a unique combination of features that are important in robot-enhanced interventions for children with NDD and are seldom supported together in existing robots used in this domain. We have compared Puffy against 21 most relevant robots that, according to the existing literature, have been used in interventions for subjects with NDD: Bobus [47], CHARLIE [7], CPAC [47], DISKCAT [47], FACE [44], Infanoid [15], IROMEC [15], Jumbo [47], KASPAR [57], Keepon [22], Kismet [11], Maestro [47], Nao [30], Paro [66], Roball [47], Robota [5], SAM [19], Tega [38], Teo [10], TREVOR [15], Troy [15]. The source for our analysis has been research publications as well additional public materials e.g., web sites and online videos, which describe the design features of these robots and on-the-field case studies with NDD children. The comparison considers the main requirements discussed in section 3 concerning the roles played by the robot in the interaction with children (Table 1) and the design of the body, the stimuli and the interaction (Table 2 ). The robots marked with a "*” have been designed since the very beginning for children with NDD (CHARLIE, DISKCAT, IROMEC, Sam, Teo, Puffy) or at least for subjects with neurological impairments (e.g., Paro). For brevity, these robots are hereinafter referred to as "specialized", while the others are called "generic". Besides offering a comparison that highlights the uniqueness of Puffy, our analysis also looks to the current state of the art from a novel perspective, shedding a light on the main design features of existing robots 
used in interventions for NDD children. Not surprisingly, Puffy - the design of which has been informed by the requirements stated in section 3 - achieves the highest scores in both tables and is the only robot that matches all parameters.

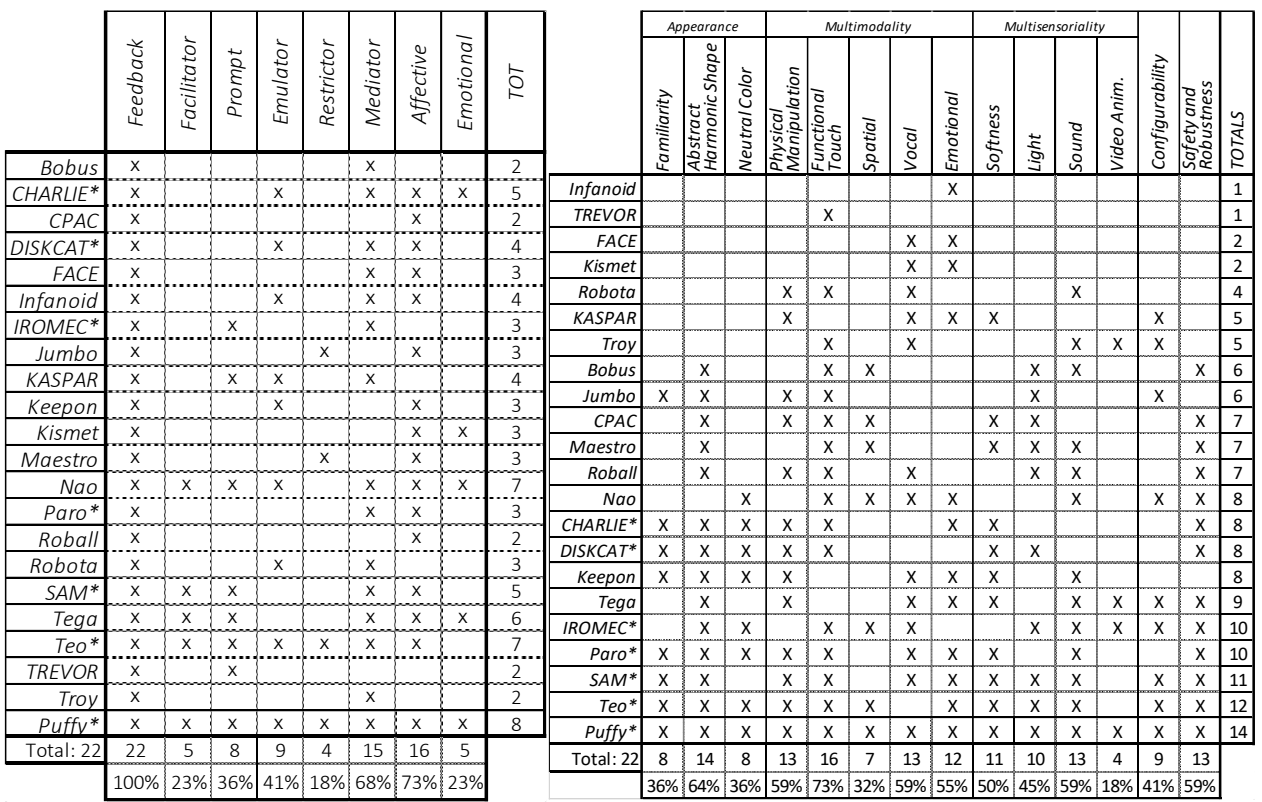

Table 1. Comparison between robots' ro- Table 2. Comparison between design features (robot nales (robot names in alphabetical order) mes ordered according to their ranking)

Concerning Table 1, all robots play as Feedback; it seems to be widely acknowledged the importance not simply of supporting interaction, i.e., providing stimuli in response to user's actions, but also of offering positive reinforcement and rewarding the children. Table 1 also shows an expected dependency between the physical capability of the robot and its roles. For example, 2 robots (Nao and Sam) meet all but one requirements, respectively Space Restrictor (Nao is static) and Emotional Agent (Teo is not equipped with emotion detection functionality). Table 1 also highlights the potential of robots to promote affection: $73 \%$ of the robots play as Affective Agent; some robots in this subset (e.g., Paro, Teo, SAM) have been explicitly designed for this role while for others (e.g. [42]) the affection power emerged only during the experimentation with the children. The second most popular role is Social Mediator ( $68 \%$ of the robots). This role is played by all specialized robots and also by 6 generic robots. Specialized robots are typically meant to be used by children under the supervision of the caregiver and all acknowledge that the interaction with the robot promotes also human-human interaction; still, the potential of robotic interaction to develop social skills in children with NDD seems to be achieved also by some generic robots, showing that it might be intrinsic in the robotic interaction paradigm per se.

Concerning Table 2, again the highest total scores $(>7)$ are assigned to all specialized robots, but this top ranked set also includes some generic robots (Nao, Roball, Maestro). The most common physical feature is the abstract non-humanoid shape (64\%) and the least common one is the integration with videos and animations (18\%). All specialized robots but IROMEC, plus 3 generic robots, are soft, accounting to $50 \%$ of the total. The most popular paradigm of interaction is Functional touch (73\%) followed by Physical Manipulation and Vocal Interaction (59\%). The latter is supported by 4 out of 7 specialized robots. There is some interest in the conversational paradigm in the NDD arena, for improving 
language and communication skills; still, we should consider that some children with NDD might be nonverbal and could not exploit the benefits of voice-based interaction. A limited number of robots $(32 \%)$ exploits spatial interaction, an interaction paradigm that is relatively new and deserves further exploration. The values of the various robots resulting from our analysis do not reflect their therapeutic or educational effectives for children with NDD and is therefore not to be interpreted as a "quality" ranking. Certainly, the design and functionalities of the robot have a significant influence in the educational and therapeutic process; still, in general we cannot state that, on the basis of our comparison, some features are more relevant than others. In addition, from our analysis we cannot draw any conclusion on how each specific design feature may influence the benefits (or limitations) for each child's experience with the robot. This is because the profound diversification of the profiles of children with NDD. Due the nature of these disorders, not all children with NDD will react exactly in the same way. Some may show more receptiveness towards some design features but discomfort to others, and with few exceptions we cannot state that some design aspects are more relevant than others. An exception is safety and robustness, which is truly a precondition for this special target and, according to our analysis, and is satisfied only by $59 \%$ of the robots ( 7 specialized robots and 6 generic ones). A rigorous comparative analysis of these aspect can be hardly derived from public material, and would require an in-depth systematic research through controlled empirical studies.

\section{PRELIMINARY EVALUATION}

\subsection{Session with NDD specialists}

After the development of the first prototype, we organized a half day workshop with 10 specialists in NDD (therapists, special educators and psychologists) to collect feedbacks on our design solutions. Beside the technical team, participants included 5 caregivers who have been cooperating with our research in the last 3 years and helped us to identify the requirements stated in section 3 , and 10 specialists with no limited experience in robotic interaction. The workshop was organized in 3 main phases. We first presented the set of requirements that informed our design; in the focus-group like session that followed we explored participants' agreements or disagreements on each requirement. We collected a general consensus on the requirements and many highlights on the user needs underlying them, as well as examples of how the current practices address such needs, their limitations, and how a robot meeting such requirements would help. In the second phase, we then demonstrated Puffy and participants played freely with Puffy and performed all the activities reported in section 4, alternating remotely controlled (first operated by the technical team, and then by themselves) and autonomous modes. In the final phase, we asked participants to fill an online questionnaire and evaluate Puffy as a whole and on each single feature, on a 4-points Likert scale, and to include comments and justifications of scores. The results were immediately available and were discussed with participants. Figure 12 reports the most salient findings.

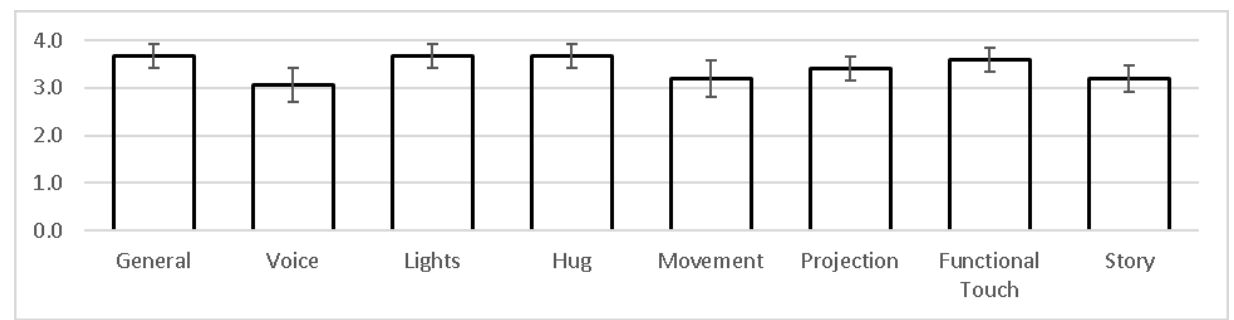

Figure 12. Preliminary evaluation: Key results of specialists' survey 


\subsection{Session with children}

One of the workshop participants invited us to bring Puffy at her primary school ${ }^{1}$ and to try it with 2 children with ASD (medium-functioning, aged 7 and 9). At the school, a big thing like Puffy couldn't go unnoticed and several students enthusiastically asked to play with it. So the robot was used among the entire class of the two ASD children (19 children, average age 6). They played with the robot for approximately one hour under the supervision of the technical team, the special educator, and the regular teacher. They were initially involved in free play, and then performed one of each type of activities described in the previous sections, interacting with Puffy in turn. The observation of this very informal experimental session indicate that Puffy was perceived as pleasant and fun by the 2 children with NDD. During the experience they showed behaviors that, according to the educator, they had very seldom manifested in the past. For example, they were not afraid by "the new thing" (which would be a typical reaction of subjects with autism) and immediately perceived it as safe, good, harmless: they were invited to be the first ones to play with the robot ("Puffy is here for you!") and immediately accepted and started touching it. When it was not their turn of interacting, they did not leave the group as it normally happens, but payed attention to the other peer' action and Puffy's reactions. For all children, Puffy acted as an extraordinary catalyzer of attention and a source of fun. Many children, leaving the session room and saying "Hello" to the robot also asked it to come back again soon.

\subsection{Discussion}

Concerning the session with specialists, the survey results show a general agreement of the potential of Puffy for children with NDD (as witnesses by the "General" bar in Figure 12). All participants declared that they would include Puffy in their therapeutic treatment.

Figure 12 shows that the light stimuli are a design feature that specialist consider as particularly promising; many of them declared that the soft lights of Puffy have the power of attracting the children as well as to relax them. The most appreciated interaction mode is tangible interaction, particularly hugging. The unique hugging characteristic of Puffy originate from its big inflatable body, and the above result suggests that inflatable shapes deserves further investigation in the NDD arena. Voice features had the lowest score; according to specialists, Puffy does not hold the capability of automatically creating conversation which are appropriate for children with NDD, who often have a weak vocabulary and make many errors. This comment was somehow expected, as our speech engine use standard speech interpretation mechanisms which are not trained to the characteristics of this target group. Among the qualitative comments reported in the survey, some offered interesting insights on risks. Some specialists observed that that in order to become a reliable element in a therapeutic or educational program, it's important that Puffy has not only attractive features but also enables mechanisms for avoiding or mitigating the emergence of undesirable situations, e.g., a child's "negative" reaction such as aggressively or stereotypes, supporting not only physical safety (of the robot and the children) but also psychological and social harmless. This problem deserves further multidisciplinary research. Addressing it requires an accurate case analysis, grounded on the current practice and on an extensive experimentation of Puffy, and would lead to the design of adaptation mechanisms in the robot behavior that are beyond the current state of the art in adaptive robotics.

In spite of its brevity and informal nature, also the session at the school supports the hypothesis that Puffy has a good potential for children with NDD. The experience also

\footnotetext{
${ }^{1}$ There are no "special education" schools in our country; children with disability attend the same school as children without disability, and are assigned to a special educator who assist them during school time.
} 
suggests that this robot might be appropriate not only in the contexts for which it was originally conceived (therapeutic centers) but also in regular schools, and for children with and without disability. This new scenario would require a further analysis to extend the set of activities currently designed for Puffy, in order to address the characteristics of broader targets and contexts. To explore the potential of Puffy also from this perspective, we are currently conducting an exploratory study at a local pre-school involving 79 children (aged 3-5) and their 15 teachers.

A final observation concerns the "fun" nature of Puffy, which at school was enhanced by some unpredictable events during remotely controlled interactions. Caregivers triggered by mistake a number of "crazy" behaviors, which were totally inconsistent with the current interaction context, but particularly funny for all children, including those with ASD. Enforcing behavioral consistency, predictability and repeatability is a fundamental requirement in the design of socially assistive robots for NDD; still, serendipity, unpredictability, and surprise could be design concepts worth to explore in relationship of this target group.

\section{CONCLUSIONS}

Our work offers an original contribution to the design of social robots for children with severe and multiple disability in the cognitive and social sphere. Puffy supports learning through play taking into account the specific characteristics and needs of this target group and is the first example of assistive robot that use inflatable materials.

Puffy exemplifies how different interaction paradigms (spatial, emotional, and tangible interaction), physical features (mobility, inflatable body, from-inside multimedia projections) and multisensory stimuli can be combined and orchestrated to create a novel robotic companion robot for children with NDD. The design of Puffy was informed by a set of requirements that distill the main results of the current state of the art in this field as well, the knowledge of a team of NDD specialists that are collaborating in our research, and the experience gained in the last 10 years in the development of assistive technology for children with NDD. These requirements can be regarded as design guidelines that can benefit researchers and developers in social assistive robotics in this domain. On the basis of these guidelines, we have provided a systematic comparison between Puffy and 21 existing robots that have been used with children in the NDD spectrum. This analysis offers a novel perspective on the current state of the art in assistive robotics for this target group. We performed two preliminary exploratory sessions with Puffy - a focus group with specialists and an activity at a primary school - which offered encouraging results. Still, Puffy has not yet been evaluated in a systematic field study, which is one of the next steps in our research agenda. Additional future actions include the improvement of the design and implementation of Puffy according to the suggestions emerged during the workshop with the specialists, particularly those related to the need of adaptation mechanism to face critical situation of children's distress or misbehavior, and improvements of the vocal interaction capability.

\section{ACKNOWLEDGMENT}

Thanks to all teachers, specialists and children from "Via Gallina" and "Munari" kindergarten schools, "Fraternità e Amicizia" and "L'abilità" therapeutic centers. 


\section{REFERENCES}

1. American Psychiatric Association, 2013. DSM 5. American Psychiatric Association.

2. Bauminger, N., 2002. The facilitation of social-emotional understanding and social interaction in high-functioning children with autism: Intervention outcomes. Journal of autism and developmental disorders, 32(4), pp.283-298.

3. Belpaeme, T., Baxter, P.E., Read, R., Wood, R., Cuayáhuitl, H., Kiefer, B., Racioppa, S., KruijffKorbayová, I., Athanasopoulos, G., Enescu, V. and Looije, R., 2012. Multimodal child-robot interaction: Building social bonds. Journal of Human-Robot Interaction, pp.33-53.

4. Best, C.M., Wilson, J.P. and Killpack, M.D., 2015, November. Control of a pneumatically actuated, fully inflatable, fabric-based, humanoid robot. In Humanoid Robots (Humanoids), 2015 IEEE-RAS 15th International Conference on (pp. 1133-1140). IEEE.

5. Billard, A., Robins, B., Nadel, J. and Dautenhahn, K., 2007. Building robota, a mini-humanoid robot for the rehabilitation of children with autism. Assistive Technology, pp.37-49.

6. Bird, G., Leighton, J., Press, C. and Heyes, C., 2007. Intact automatic imitation of human and robot actions in autism spectrum disorders. Proc. Royal Society of London B: Biological Sciences, 274(1628), pp.3027-3031.

7. Boccanfuso, L. and O'Kane, J.M., 2011. CHARLIE: An adaptive robot design with hand and face tracking for use in autism therapy. International journal of social robotics, pp.337-347.

8. Bonarini, A., 2016. Can my robotic home cleaner be happy? Issues about emotional expression in non-bio-inspired robots. Adaptive Behavior, 24(5), pp.335-349.

9. Bonarini, A., Clasadonte, F., Garzotto, F. and Gelsomini, M., 2015, June. Blending robots and full-body interaction with large screens for children with intellectual disability. In Proc. 14th International Conference on Interaction Design and Children (pp. 351-354). ACM.

10. Bonarini, A., Garzotto, F., Gelsomini, M., Romero, M., Clasadonte, F. and Yilmaz, A.N.Ç., 2016, August. A huggable, mobile robot for developmental disorder interventions in a multimodal interaction space. In Robot and Human Interactive Communication (RO-MAN), 2016 25th IEEE International Symposium on (pp. 823-830). IEEE.

11. Breazeal, C. and Scassellati, B., 1999. A context-dependent attention system for a social robot. rn, 255, p.3.

12. Breazeal, C., 1998, July. A motivational system for regulating human-robot interaction. In Aaai/iaai (pp. 54-61).

13. Brodin, J., 1999. Play in Children with Severe Multiple Disabilities: play with toys-a review. International Journal of Disability, Development and Education, 46(1), pp.25-34.

14. Bruner, J.S., Jolly, A. and Sylva, K. 1976. Play: Its role in development and evolution. Journal of Personality Assessment.

15. Cabibihan, J.J., Javed, H., Ang, M. and Aljunied, S.M., 2013. Why robots? A survey on the roles and benefits of social robots in the therapy of children with autism. International journal of social robotics, 5(4), pp.593-618.

16. Chella, A., Barone, R.E., Pilato, G. and Sorbello, R., 2008, April. An Emotional Storyteller Robot. In AAAI Spring Symposium: Emotion, Personality, and Social Behavior (pp. 17-22).

17. Ciolek, T.M. and Kendon, A., 1980. Environment and the spatial arrangement of conversational encounters. Sociological Inquiry, 50(3-4), pp.237-271.

18. Clark, T., Feehan, C., Tinline, C. and Vostanis, P., 1999. Autistic symptoms in children with attention deficit-hyperactivity disorder. European child \& adolescent psychiatry, pp.50-55.

19. Colombo, S., Garzotto, F., Gelsomini, M., Melli, M. and Clasadonte, F., 2016, June. Dolphin Sam: A Smart Pet for Children with Intellectual Disability. In Proceedings of the International Working Conference on Advanced Visual Interfaces (pp. 352-353). ACM.

20. Dautenhahn, K. and Werry, I., 2004. Towards interactive robots in autism therapy: Background, motivation and challenges. Pragmatics \& Cognition, 12(1), pp.1-35.

21. Dautenhahn, K., Werry, I., Salter, T. and Boekhorst, R.T., 2003, July. Towards adaptive autonomous robots in autism therapy: Varieties of interactions. In Computational Intelligence in Robotics and Automation, 2003. Proceedings. 2003 IEEE International Symposium on (Vol. 2, pp. 577-582). IEEE. 
22. Den Brok, W.L.J.E. and Sterkenburg, P.S., 2015. Self-controlled technologies to support skill attainment in persons with an autism spectrum disorder and/or an intellectual disability: a systematic literature review. Disability and Rehabilitation: Assistive Technology, pp.1-10.

23. Diehl, J.J., Schmitt, L.M., Villano, M. and Crowell, C.R., 2012. The clinical use of robots for individuals with autism spectrum disorders: A critical review. Research in autism spectrum disorders, 6(1), pp.249-262.

24. Dunst, C.J., Hamby, D.W., Trivette, C.M., Prior, J. and Derryberry, G., 2013. Vocal Production of Young Children with Disabilities during Child-Robot Interactions. Social Robots Research Reports, Number 5. Orelena Hawks Puckett Institute.

25. Feil-Seifer, D. and Matarić, M.J., 2011. Socially assistive robotics. IEEE Robotics \& Automation Magazine, 18(1), pp.24-31.

26. Ferrara, C. and Hill, S.D., 1980. The responsiveness of autistic children to the predictability of social and nonsocial toys. Journal of autism and developmental disorders, pp.51-57.

27. Ferrari, E., Robins, B. and Dautenhahn, K., 2009, September. Therapeutic and educational objectives in robot assisted play for children with autism. In Robot and Human Interactive Communication, 2009. RO-MAN 2009. The 18th IEEE International Symposium on (pp. 108-114). IEEE.

28. Garzotto, F. and Bordogna, M., 2010, June. Paper-based multimedia interaction as learning tool for disabled children. In Proceedings of the 9th international Conference on Interaction Design and Children (pp. 79-88). ACM.

29. Garzotto, F., 2014. Interactive storytelling for children: a survey. International Journal of Arts and Technology, 7(1), pp.5-16.

30. Gillesen, J.C., Barakova, E.I., Huskens, B.E. and Feijs, L.M., 2011, June. From training to robot behavior: Towards custom scenarios for robotics in training programs for ASD. In Rehabilitation Robotics (ICORR), 2011 IEEE International Conference on (pp. 1-7). IEEE.

31. Giullian, N., Ricks, D., Atherton, A., Colton, M., Goodrich, M. and Brinton, B., 2010, October. Detailed requirements for robots in autism therapy. In Systems Man and Cybernetics (SMC), 2010 IEEE International Conference on (pp. 2595-2602). IEEE.

32. Groden, J., Cautela, J., Prince, S. and Berryman, J., 1994. The impact of stress and anxiety on individuals with autism and developmental disabilities. In Behavioral issues in autism (pp. 177194). Springer US.

33. Grove, N., 2012. Using storytelling to support children and adults with special needs: transforming lives through telling tales. Routledge.

34. Hall, E.T., 1966. The hidden dimension.

35. Hoa, T.D. and Cabibihan, J.J., 2012, November. Cute and soft: baby steps in designing robots for children with autism. In Proceedings of the Workshop at SIGGRAPH Asia (pp. 77-79). ACM.

36. Kasari, C., Gulsrud, A.C., Wong, C., Kwon, S. and Locke, J., 2010. Randomized controlled caregiver mediated joint engagement intervention for toddlers with autism. Journal of autism and developmental disorders, 40(9), pp.1045-1056.

37. Kinoe, Y. and Mizuno, N., 2016, July. Dynamic Characteristics of the Transformation of Interpersonal Distance in Cooperation. In International Conference on Human Aspects of IT for the Aged Population (pp. 26-34). Springer International Publishing.

38. Kory Westlund, J., Lee, J.J., Plummer, L., Faridi, F., Gray, J., Berlin, M., Quintus-Bosz, H., Hartmann, R., Hess, M., Dyer, S. and Dos Santos, K., 2016, March. Tega: A social robot. In The Eleventh ACM/IEEE International Conference on Human Robot Interaction (pp. 561-561). IEEE Press.

39. Kozima, H., Michalowski, M.P. and Nakagawa, C., 2009. Keepon. International Journal of Social Robotics, 1(1), pp.3-18.

40. Kozima, H., Nakagawa, C. and Yasuda, Y., 2007. Children-robot interaction: a pilot study in autism therapy. Progress in Brain Research, 164, pp.385-400.

41. Kuypers, L., 2011. The zones of regulation. San Jose: Think Social Publishing.

42. Park, H.W., Gelsomini, M., Lee, J.J. and Breazeal, C., 2017, March. Telling Stories to Robots: The Effect of Backchanneling on a Child's Storytelling. In Proceedings of the 2017 ACM/IEEE International Conference on Human-Robot Interaction (pp. 100-108). ACM. 
43. Majidi, C., 2014. Soft robotics: a perspective-current trends and prospects for the future. Soft Robotics, 1(1), pp.5-11.

44. Mazzei, D., Billeci, L., Armato, A., Lazzeri, N., Cisternino, A., Pioggia, G., Igliozzi, R., Muratori, F., Ahluwalia, A. and De Rossi, D., 2010, September. The face of autism. In RO-MAN, 2010 IEEE (pp. 791-796). IEEE.

45. Mead, R. and Matarić, M.J., 2016. Perceptual models of human-robot proxemics. In Experimental Robotics (pp. 261-276). Springer International Publishing.

46. Mehrabian, A., 1972. Nonverbal communication. Transaction Publishers.

47. Michaud, F., Duquette, A. and Nadeau, I., 2003, October. Characteristics of mobile robotic toys for children with pervasive developmental disorders. In Systems, Man and Cybernetics, 2003. IEEE International Conference on (Vol. 3, pp. 2938-2943). IEEE.

48. Mori, M., MacDorman, K.F. and Kageki, N., 2012. The uncanny valley [from the field]. IEEE Robotics \& Automation Magazine, 19(2), pp.98-100.

49. Mumm, J. and Mutlu, B., 2011, March. Human-robot proxemics: physical and psychological distancing in human-robot interaction. In Proceedings of the 6th international conference on Human-robot interaction (pp. 331-338). ACM.

50. Phillips, B. and Zhao, H., 1993. Predictors of assistive technology abandonment. Assistive technology, 5(1), pp.36-45.

51. Pierno, A.C., Mari, M., Lusher, D. and Castiello, U., 2008. Robotic movement elicits visuomotor priming in children with autism. Neuropsychologia, 46(2), pp.448-454.

52. Qi, R., Lam, T.L. and Xu, Y., 2014, May. Mechanical design and implementation of a soft inflatable robot arm for safe human-robot interaction. In Robotics and Automation (ICRA), 2014 IEEE International Conference on (pp. 3490-3495). IEEE.

53. Rabbitt, S.M., Kazdin, A.E. and Scassellati, B., 2015. Integrating socially assistive robotics into mental healthcare interventions: Applications and recommendations for expanded use. Clinical psychology review, 35, pp.35-46.

54. Ricks, D.J. and Colton, M.B., 2010, May. Trends and considerations in robot-assisted autism therapy. In Robotics and Automation (ICRA), 2010 IEEE International Conference on (pp. 4354-4359). IEEE.

55. Rios-Martinez, J., Spalanzani, A. and Laugier, C., 2015. From proxemics theory to sociallyaware navigation: A survey. International Journal of Social Robotics, 7(2), pp.137-153.

56. Robins, B., Amirabdollahian, F. and Dautenhahn, K., 2013. Investigating child-robot tactile interactions: A taxonomical classification of tactile behaviour of children with autism towards a humanoid robot. In The sixth international conference on Advances in Computer-Human Interactions (ACHI) (pp. 89-94).

57. Robins, B., Dautenhahn, K. and Dickerson, P., 2009, February. From isolation to communication: a case study evaluation of robot assisted play for children with autism with a minimally expressive humanoid robot. In Advances in Computer-Human Interactions, 2009. ACHI'09. Second International Conferences on (pp. 205-211). IEEE.

58. Robins, B., Dautenhahn, K. and Dubowski, J., 2006. Does appearance matter in the interaction of children with autism with a humanoid robot?. Interaction studies, 7(3), pp.509-542.

59. Robins, B., Dautenhahn, K., Ferrari, E., Kronreif, G., Prazak-Aram, B., Marti, P., Iacono, I., Gelderblom, G.J., Bernd, T., Caprino, F. and Laudanna, E., 2012. Scenarios of robot-assisted play for children with cognitive and physical disabilities. Interaction Studies, pp.189-234.

60. Robins, B., Dautenhahn, K., Te Boekhorst, R. and Billard, A., 2005. Robotic assistants in therapy and education of children with autism: can a small humanoid robot help encourage social interaction skills?. Universal Access in the Information Society, 4(2), pp.105-120.

61. Robins, B., Otero, N., Ferrari, E. and Dautenhahn, K., 2007, August. Eliciting requirements for a robotic toy for children with autism-results from user panels. In Robot and Human interactive Communication, 2007. RO-MAN 2007. The 16th IEEE International Symposium on (pp. 101106). IEEE.

62. Rommelse, N.N., Franke, B., Geurts, H.M., Hartman, C.A. and Buitelaar, J.K., 2010. Shared heritability of attention-deficit/hyperactivity disorder and autism spectrum disorder. European child \& adolescent psychiatry, 19(3), pp.281-295. 
63. Salter, T., Davey, N. and Michaud, F., 2014, August. Designing \& developing QueBall, a robotic device for autism therapy. In Robot and Human Interactive Communication, 2014 RO-MAN: The 23rd IEEE International Symposium on (pp. 574-579). IEEE.

64. Scassellati, B., Admoni, H. and Matarić, M., 2012. Robots for use in autism research. Annual review of biomedical engineering, 14, pp.275-294.

65. Shamsuddin, S., Yussof, H., Ismail, L., Hanapiah, F.A., Mohamed, S., Piah, H.A. and Zahari, N.I., 2012, March. Initial response of autistic children in human-robot interaction therapy with humanoid robot NAO. In Signal Processing and its Applications (CSPA), 2012 IEEE 8th International Colloquium on (pp. 188-193). IEEE.

66. Shibata, T., Mitsui, T., Wada, K. and Tanie, K., 2002. Subjective evaluation of seal robot: Parotabulation and analysis of questionnaire results. Journal of Robotics and Mechatronics, 14(1), pp.13-19.

67. Short, E., Swift-Spong, K., Greczek, J., Ramachandran, A., Litoiu, A., Grigore, E.C., Feil-Seifer, D., Shuster, S., Lee, J.J., Huang, S. and Levonisova, S., 2014, August. How to train your dragonbot: Socially assistive robots for teaching children about nutrition through play. In Robot and Human Interactive Communication, 2014 RO-MAN: The 23rd IEEE International Symposium on (pp. 924-929). IEEE.

68. Stiehl, W.D., Lieberman, J., Breazeal, C., Basel, L., Lalla, L. and Wolf, M., 2005, August. Design of a therapeutic robotic companion for relational, affective touch. In Robot and Human Interactive Communication, 2005. ROMAN 2005. IEEE International Workshop on (pp. 408415). IEEE.

69. Voisembert, S., Riwan, A., Mechbal, N. and Barraco, A., 2011, May. A novel inflatable robot with constant and continuous volume. In Robotics and Automation (ICRA), 2011 IEEE International Conference on (pp. 5843-5848). IEEE.

70. Wilson, M., 2002. Six views of embodied cognition. Psychonomic bulletin \& review, pp.625636.

71. Valoriani M., Bartoli L., Garzotto F., Gelsomini M., Oliveto L, 2014. Designing and evaluating touchless playful interaction for ASD children. In Proceedings of the 2014 conference on Interaction design and children (IDC '14). ACM, New York, NY, USA, 17-26.

72. Valoriani M, Bartoli L., Corradi C, Garzotto F. 2013 Motion-based Touchless Interaction for Autistic Children's learning. In Proc. ACM Interaction Design and Children (IDC) 2013, 53-44 